\title{
Blockchain Technology Helps the Development of Meteorological Informatization
}

\author{
Guanghui Wang* \\ Center for Meteorological data Analysis and Application, Chinese Academy of Meteorological Sciences, Beijing 100081, China \\ *Corresponding author: ghwang@cma.gov.cn
}

Received July 13, 2019; Revised August 22, 2019; Accepted September 05, 2019

\begin{abstract}
With the impact of the Internet, the application of information technology promotes the continuous change and development of meteorological field. Based on the intrinsic motivation of the development and change of meteorological field, the impetus of blockchain technology to meteorological informatization is discussed in this paper. Firstly, the application status and development opportunities of information technology in meteorological field are analyzed. Then, the new requirements of meteorological information business development driven by information technology are presented. Finally, the paper deals with the role of blockchain technology in promoting the modernization of meteorological information and points out that the combination of blockchain technology with artificial intelligence and big data will further develop and improve meteorological information technology in many aspects.
\end{abstract}

Keywords: blockchain, big data, artificial intelligence, cloud computing, meteorological information

Cite This Article: Guanghui Wang, "Blockchain Technology Helps the Development of Meteorological Informatization.” Journal of Business and Management Sciences, vol. 7, no. 3 (2019): 112-120. doi: 10.12691/jbms-7-3-2.

\section{Introduction}

The rapid development and deep application of electronic communication technology in the second industrial revolution and computer and a series of related technologies in the third industrial revolution promoted the formation of meteorological science and the establishment of meteorological business. Information was extracted and analyzed from collected data, then meteorological information service was provided. Therefore, the third industrial revolution (i.e. the information technology revolution) and the fourth industrial revolution (i.e. the Internet revolution) proposed by many scholars have greatly promoted the development of meteorological disciplines and business. Without information technology, there will be no meteorological modernization. Information technology not only supports modern meteorological business, but also promotes the rapid development of meteorological business and scientific research. At the same time, the ideas, concepts and methods that have emerged in the tide of information technology for decades have gradually infiltrated into the ideology of meteorological business and management personnel, imperceptibly improving their thinkingmethods and working modes. Intensification, work collaboration, process optimization, scientific management, top-level design, architecture planning, knowledge management and so on have gradually become the specific content of meteorological department's work. However, with the use of new information technologies such as cloud computing, artificial intelligence, and big data, new requirements have been put forward for the development of meteorological information technology. For example, the storage of massive meteorological data, the value ownership of meteorological data, the sharing of data resources, the quality traceability of meteorological data, and the security of meteorological data will bring new problems to meteorological information services. These problems will hinder the use and promotion of information technology in meteorological field. The blockchain with the spirit of coordination and cooperation is inherent. Especially, the "public chain" is characterized by openness, non-tampering, traceability, transnational and decentralization, which brings opportunities for solving these problems. Based on the basic principles and characteristics of blockchains, this paper discusses the role of blockchains in promoting meteorological information technology [1].

\section{Fundamentals, Characteristics, and Development Trend of Blockchain}

Blockchain technology, as a computer underlying technology, has attracted more and more attention from the rise of Bitcoin. It is being developed vigorously and applied in various areas. 


\subsection{The Basics and Characteristics of Blockchain Technology}

\subsubsection{Concepts}

Blockchains originate from Bitcoin. Document [2] elaborates the architecture concept of e-cash system based on P2P network technology, encryption technology, timestamp technology, blockchain technology, and so on, which marks the birth of Bitcoin. In the process of formation of bitcoin, blocks are storage units, recording all the exchange information of each block node in a certain time, linking each block through random hash (also known as hash algorithm). The latter contains the hash value of the former block. With the expansion of information exchange, block connection one by one constitutes a blockchain [3].

\subsubsection{Technical Characteristics}

From the formation process, blockchain technology has the following characteristics [4]:

(1) Decentralization. Traditional database technology architecture is private and centralized. The key to solve value transfer in this architecture is to solve the problem of mutual trust. Blockchain technology does not rely on third-party management agencies or hardware facilities. There is no central control. Through distributed accounting and storage, each node achieves information self-verification, transmission, and management, which is the most prominent and essential feature of blockchain technology.

(2) Distributed storage, open and transparent. Blockchain technology is based on open source. Apart from the encrypted private information of all parties, the data of blockchain is open to everyone. Anyone can query blockchain data and develop related applications through open interfaces. The information of the whole system is highly transparent. This approach solves the problem of information islands in traditional information systems. At the same time, computing nodes can be deployed on any platform.

(3) Consensus mechanism. Based on consensus specifications and protocols (like bitcoin hashing algorithm and other data algorithms), the whole blockchain system does not rely on third parties, and all nodes can automatically and safely verify and exchange data within the system without any human intervention. The biggest subversion of blockchain theory lies in the new credit formation mechanism.

(4) Safety. Through the form of distributed database, each participating node can get a copy of the complete database. As long as $51 \%$ of all data nodes cannot be mastered, it is impossible to manipulate and modify network data arbitrarily, which makes the blockchain itself relatively safe and avoids data modification. Blockchains are based on timestamps to form non-tamper and nonforgeable databases. The addition of blocks (complete history) and chains (complete validation) forms a timestamp (traceable complete history). Timestamp stores all transaction history performed in the network, provides retrieval and search functions for each data, and achieves traceability function by means of blockchain structure.

(5) Information encryption and anonymity. Unless there are legal requirements, technically speaking, the identity information of each block node does not need to be disclosed or verified. Information transmission is anonymous. Only those who have the private key can open their own "wallet". Blockchains are open on the network and can be queried in each offline Bitcoin wallet data.

\subsection{Application Status and Development Trend of Blockchain Technology}

\subsubsection{Application Status}

At present, as a new technology industry with national strategic development significance, blockchain technology has been paid more and more attention all over the world. Many large multinational companies have laid out blockchain industry, and this new technology has been involved in more and more industries. The high-tech industry continuously excavates the blockchain technology, devotes itself to building the ecosystem which integrates the technology, develops and runs the system, promotes the integration and innovation of the blockchain and the existing technology, and provides the bottom distributed application protocol for the commercial operation. Assist in the transformation of the existing operational model. Blockchain technology is a fully open source system, the principle and code are open, and in the course of running under the supervision of all nodes in the system. The application scenarios of blockchain technology include financial services, supply chain management, cultural entertainment, intelligent manufacturing, social welfare, education and employment, meteorology and so on. There are three levels: blockchain 1.0, blockchain 2.0 and blockchain 3.0. Blockchain 1.0 refers to the application of block chain in virtual digital money market such as money transfer and payment system. Because of the low cost of issuance and circulation of the virtual digital currency and the convenience and transparency of the economic transaction, the blockchain, which is the support technology of the virtual digital currency, has naturally become the preferred technology of issuing digital currency in various countries. Blockchain 2.0 mainly refers to the application of blockchain in financial markets such as securities registration, futures, loans, bills and so on, mainly in four fields: Internet finance, financial market infrastructure construction, paper market, P2P network loan and so on. Blockchain 3.0 extends the technology to a wider application market, permeates all aspects of human life, and realizes information sharing around the world based on trust mechanism. Its greatest contribution is to reduce the financial risk, not only the transaction costs have been reduced, but also to a certain extent to avoid the subjective factors of the impact of data, improve the objective notarization of value measurement.

\subsubsection{Development Trend}

Generally speaking, the application research of blockchain has transited from the original pure digital currency to the broader financial industry and extended into many areas of society.

1) Blockchains will no longer be limited to providing digital currency trading platform but will further test the background procedures of digital asset trading to improve transaction efficiency, reduce costs and enhance transaction 
security. In the future, the research focus of digital currency + blockchain is the deep development of Distributed Accounting technology.

2) Blockchains based on decentralized distributed structure can complete de-trust transactions between nodes with zero mediation participation. Blockchain technology is introduced into the basic system to maintain customer information security and reduce the cost of legal supervision and the incidence of financial crimes. People will explore the application of "Blockchain + Internet of Things" and "Blockchain + Artificial Intelligence". The Internet of Things improves the interconnection and interoperability between devices by means of Blockchain technology, reduces the cost of connection between devices, and improves the trust and security between users, devices and networks. Artificial intelligence will be strengthened by using Blockchain, which will enhance the credibility, accuracy and security of information.

3) In the future, the blockchain will be more and more applied to all aspects of social life. Firstly, the application of blockchains in the field of big data; secondly, the application of blockchains in the field of communication; thirdly, the application of blockchains in the field of asset management; and fourthly, the application of blockchains in the field of logistics supply chain. In addition, there are many other applications to be further explored and studied. For example, how to use blockchain technology to solve the trust in the field of climate change and environmental protection, the transaction of meteorological data, meteorological insurance services, maintenance of meteorological equipment, and early warning release of meteorological information. In addition, the fields of existence certification, authentication, forecasting market, intelligent contract, e-commerce, file storage and so on will gradually become the frontier topics of blockchain application research.

\section{Information Technology and Meteorological Informatization}

Informatization has entered a new stage of comprehensive penetration, cross-border integration, accelerating innovation and leading development, creating a new space for human beings and triggering new changes in social production. Meteorological information affecting all aspects of society, economy and life has become an important data resource for government social governance and natural disaster management. Driving meteorological modernization by meteorological informatization and building intelligent meteorology are important measures to implement innovation-driven and national information development strategy. The development of information technology will strongly promote the development of meteorological informatization.

\subsection{The Great Impact of Information Technology on Traditional Meteorological Information Business}

The meteorological information service has undergone several changes in the development of several decades. It has roughly experienced the design, construction, operation, maintenance and service of the basic information technology resources (i.e., communication resources, computing resources, data resources, storage resources and network resources) of meteorological departments. With the emergence and rapid popularization of cloud computing, it is possible to provide high-quality services and low-cost use of IT basic resources such as computing, storage and network. The era of self-construction, selfmanagement and self-use of IT basic resources by various units has gone forever. Under this background, the responsibilities and work areas of IT infrastructure resources construction, management, maintenance and service in various departments' IT business are being squeezed by big data centers and cloud computing relentlessly. For meteorological departments, intensive management of data resources and high-performance computing resources constitutes an abundant data resource pool, so that all operations are centered on data, instead of continuing the data developed gradually in the 1980s and 1990s around computing resources and the working mode of database blooming everywhere, which is the consensus and established policy of meteorological departments. Therefore, it is a general trend to replace the data management system of meteorological departments in provinces, cities and counties by a limited number of large-scale data centers. Especially the introduction of artificial intelligence and blockchain technology opens up a new way for the development of meteorology. Therefore, the traditional meteorological information business, which mainly focuses on the management of basic resources construction and data services, is facing a huge impact of new information technology.

\subsubsection{Applications of Artificial Intelligence in Meteorological Field}

As early as the 1980s, China Meteorological Administration began to study the application of Artificial Intelligence (AI). The meteorological department also carried out further experiments on the application of expert nervous system in meteorological forecasting. Experts used the adaptive and fault-tolerant functions and characteristics of artificial neural network to make up for the shortcomings of expert system and effectively improve the success rate of disaster forecasting.

In the 1990s, experts from meteorological departments gradually focused on machine learning and computer simulation of specific meteorological hazards, and began to pay attention to some important basic work, such as automatic recognition of basic forms of troughs, ridges, fronts, high and low pressure centers, typhoon cloud systems and so on. With the advent of the Internet era, some Internet companies began to use artificial intelligence technology to provide short-term accurate meteorological services and achieved good results. However, these short-term forecasting results are limited to the application of daily data used by meteorological departments, and the computing resources used in forecasting processing are also very limited, not to mention the classical theories and algorithms such as weather dynamics. Instead, the accuracy of shortterm forecasting is gradually improved by training the input data. Thus, artificial intelligence technology will play an increasingly important role in the field of meteorology. 
1) Applications of AI in Meteorological Data Processing

AI has always been paid attention to in meteorological data processing, and new technologies have been emerging, such as: using conditional generation antagonistic neural network to remove radar clutter; using convolutional neural network to identify rainfall areas on satellite images; using cognitive learning method to copy remote sensing inversion and data assimilation results, so that the current numerical model system can be well assimilated quickly. Increased satellite remote sensing data. It shows that AI can save a lot of computing resources. Compared with assimilation variational algorithm, AI algorithms have higher computing efficiency (e.g.[5]).

2) Applications of AI in Weather Forecast

AI technology has been widely used in Environmental Meteorological prediction, factor probability prediction, typhoon ocean, extreme disasters and agricultural meteorology. The AI algorithms used include random forest classification technology, deep neural network, convolution neural network, convolution long-short memory neural network, parametric and non-parametric model coupling method, regression forest model and so on. The use of these methods has achieved remarkable results in weather forecasting operations (e.g.[6]).

3) Applications of AI in Weather and Climate Analysis

Storm characteristics: using self-organizing neural network method to distinguish atmospheric environmental parameters between tornadoes and non-tornadoes; using support vector machine (SVM) to classify and analyze real-time storms and climate.

Weather feature recognition: using in-depth learning to identify extreme weather in climate patterns, i.e. using supervised convolution structure classification method to identify and track tropical cyclones, atmospheric rivers and frontal systems; using unsupervised in-depth learning to "generate antagonistic neural networks" to analyze and express various spatial structures of atmospheric data, useful prediction information can be extracted more effectively. Automatic recognition and classification of fronts by deep learning algorithm and convolutional neural network (e.g. [7]).

In the aspect of atmospheric circulation and local weather, deep learning is used to study climate ultra-high resolution downscaling, dictionary learning is introduced into image ultra-high resolution, and the relationship between dictionary learning and ultra-high resolution deep learning is used to effectively reduce precipitation scale in local area.

\subsubsection{Applications of Big Data in Meteorological Field}

Meteorology is closely related to economic development and social life. Meteorological departments are high-tech departments which have accumulated a large amount of data. There is no doubt that these data are valuable resources and wealth. To fully tap the value of these resources and make good use of these resources, more accurate and personalized meteorological service products can be produced. Therefore, it is very necessary to realize and develop big meteorological data, which includes ground observation, satellite, radar and data prediction products. Big meteorological data accord with the "4V" characteristics of big data (e.g.[8]), such as "Volume data is large", "Variety data type is many", "Velocity data processing speed" and "Value is scarce". In the era of big data, the improvement of fault tolerance rate is no longer harsh on the measurement accuracy of initial data. Simple algorithms for big data are more effective than complex algorithms for small data. Simple algorithms lead to the limitation of accumulated errors, which will lead to a new revolution in the field of meteorology, and the timeliness of weather forecasting will become better and better. For a long time, meteorologists have been making efforts to digitalize the observation, prediction and scientific research. A weather process cannot be repeated in the past, but the recorded data are indeed permanently preserved for later research and exploration. As far as meteorological data itself is concerned, we can get the meteorological data of the past several thousand years, tens of thousands of years or even more from the known data simulation. We can also predict the meteorological environment after many years through these data. Of course, other non-meteorological factors need to be digitized. For example, we often use "teleconnection" and "mode coupling" operation methods to seek the correlation between meteorological elements and meteorological and other things. The basic characteristics of big data processing are: (1) sampling analysis is out of date in the era of big data; the object of people's analysis is not sampling data, but all data, that is, "sample = population"; (2) the obsession with accuracy is the product of the era of lack of information and simulation; only by accepting inaccuracy can we open a window that has never been touched; (3) knowing what is enough; you don't have to know why. In the era of big data, we don't have to know the reasons behind the phenomena but let the data "voice" itself.

Big meteorological data is the basis of the development of meteorological science. These multi-dimensional and high-quality scientific data play an important role in global climate change research. With the development of advanced observation technology and the rapid increase of scientific data, the understanding of physical, chemical and biological processes of climate system has been deepened; the understanding of climate and its change mechanism has been expanded; the development of climate system model has been promoted; the accuracy of climate prediction has been improved; and the impact of human activities on climate has been demonstrated. In recent two years, in the field of atmospheric science, the research of big scientific data has become more and more in-depth. Compared with the past, the research on big data shows the following characteristics: (1) less attention to solidified demand, more attention to exploratory demand; (2) less conceptual analysis, more summative analysis at the implementation level; (3) less traditional data processing, more analysis of data mining; (4) less single meteorological data analysis, more cross-cutting analysis, more analysis of domain comprehensive data; (5) the big data platform is more and more centralized, but the application coverage is more and more extensive. At the same time, how to manage and mine these big data is a challenge. Big data management needs huge storage space, fast transmission network and perfect website construction service system. It needs to strengthen the fusion processing of ground-based, space-based and space-based observation data, promote the research and development 
of data application, and promote the application of big data in various fields.

In the information society, data is becoming more and more important resources, especially in the era of big data, which is more obvious. The main application of big meteorological data in weather-related industries is meteorological data combined with industry data analysis to get the changing rules of events and some forecasts for the future. The application of big meteorological data in various industries gradually presents the following trends:

(1) Customization. Weather-related industries pay attention to different weather data points because of their different service or sales scope, such as: air-conditioning industry pays attention to temperature change, shipping pays attention to whether there is rain, snow, lightning or wind direction change; even if the focus is the same, the weight proportion of each data in the whole data type is different, which needs to be combined with the characteristics of each industry. Points provide customized services for them.

(2) Commercialization. As early as more than a decade ago, meteorological brokers appeared to provide services to weather-related industries, to ensure the safety of people's lives and property, and to provide certain guarantees for businesses to pursue their maximum interests. At this time, meteorological data has shown certain commodity characteristics. In the era of big data, the characteristics of commercialization are becoming more and more obvious, which promotes the stable and healthy development of commodity economy related to weather.

(3) Diversification. There are various industries related to meteorological data, providing customized services according to the different meteorological data concerned. With more and more industries being excavated to correlate with meteorological data, big meteorological data need to provide more and more diversified services. The realization and development of big meteorological data is of great significance for ensuring people's life safety and wealth.

\subsubsection{Applications of Blockchain Technology in Meteorological Field}

Blockchain technology has become an increasingly concerned computer underlying technology, and its application in various fields is developing vigorously. Now it is playing an increasingly important role in the field of meteorology. The application scenarios of blockchain technology in the field of meteorology are as follows (e.g.[9]):

(1) On the topic of climate change, blockchain technology is considered to be able to effectively solve the trust problems of various countries and organizations in the fields of climate change and environmental protection.

(2) In the field of meteorological data transaction, the weather data industry is facing the challenge of data sources and accuracy. Traceability and untouchable modification of blockchains can greatly reduce the risk of applying meteorological data and products.

(3) In terms of meteorological insurance services, there are risks of meteorological disasters in agricultural planting, urban transportation and construction facilities. Meteorological insurance can reduce the economic losses caused by meteorological disasters. Weather insurance has some problems in the process of claim settlement, such as difficulty in obtaining evidence and tedious process of payment. Blockchain may play an important role.

(4) In the maintenance of meteorological equipment, all parties involved in equipment operation and maintenance and intelligent observation equipment are connected to the blockchain network by using the characteristics of high autonomy and data security of blockchain technology. After equipment failure, all parties are informed at the same time and record the whole process of maintenance to ensure consistency and un-alterability of information. Compared with the current "serial" business processes of all parties, the response speed is faster.

(5) In the issuance of meteorological early warning information, the characteristics of blockchain technology, such as tamper-proof, traceability, safety and reliability, can well solve the existing problems in the issuance of meteorological early warning information.

\subsubsection{Opportunities and Challenges Brought by Information Technology to Meteorological Departments}

Meteorological information business has undergone several changes in the development of several decades. At present, it roughly refers to the design, construction, operation, maintenance and service of basic information technology resources (i.e., communication resources, computing resources, data resources, storage resources and network resources) of meteorological departments. With the emergence and rapid popularization of cloud computing, it is possible to provide high-quality services and low-cost use of IT basic resources such as computing, storage and network. The era of self-construction, selfmanagement and self-use of IT basic resources by various units has gone forever. In this context, the IT business of provincial and local meteorological departments is facing the relentless squeeze of big data centers and cloud computing in the fields of IT infrastructure resources construction, management, maintenance and service. Therefore, the traditional meteorological information business, which mainly focuses on the management of basic resources construction and data services, is facing severe challenges. Because of the extensive popularization and deepening of information technology, the development of meteorological cause and the progress of society, new problems, new demands and new challenges are constantly emerging. As far as meteorological information service departments are concerned, there are mainly the following development opportunities:

1) Development of High-Quality Meteorological Data and Basic Data Products

High quality meteorological data and basic data products are the fundamental basis of meteorological operations and meteorological scientific research. Unlike the problems of communication, processing, storage and other resources, the guarantee of data quality and the development of basic data products are core competencies, which cannot be really solved with simple investment. After the basic resources problems such as weak communication ability, limited detection means, lack of processing and storage capacity have been solved one by 
one with the large-scale construction over the years, the problems of data quality and production capacity of data products have gradually become prominent, and become the shortcomings of capacity-building of meteorological departments.

2) Standardization and Integration of large-scale Business System

Intensive integration of infrastructure resources and business systems is very important. Intensive integration of business systems is particularly important, because the large-scale "partial orderly, overall disorderly" business construction in recent ten years has resulted in numerous business systems, a large number of duplication of business functions and unprecedented complexity of business processes. Convergence does not actually touch the ultimate goal of breaking down data barriers, merging similar functions and optimizing business processes. Therefore, if we want to achieve the desired goal, we must follow the relevant business standards and norms, implement and ultimately complete the intensive integration of these many business systems.

3) Cloud/Client Transformation of Business System

Cloud computing is the main form of existence and application of basic resources, data resources and application resources in the future, which is the result of social and technological development and the consensus of the industry (including meteorological departments). The conclusion that future meteorological operations will mainly operate in the "cloud end" has been widely accepted, although whether the carrier is public cloud, private cloud or industry cloud is not yet clear. Therefore, it is an inevitable trend for business systems to be cloud-based/client-based.

4) Business Operation and Maintenance Based on the Whole Process

Cloud computing provides high-quality and low-cost services for basic resources. It combines with the ubiquitous and efficient Internet (including mobile interconnection), which makes it possible for meteorological departments to pursue intensive integration of business systems and highlevel operation and maintenance of the whole process. Current business processes are segmented and localized, resulting in poor signaling, slow response, mutual prevarication and efficiency. Low and other drawbacks will gradually become history with the emergence of the overall operation and maintenance of the whole process this is where cloud computing has a far-reaching impact on the meteorological business system.

\subsection{Prospect of Meteorological Information Business in the Future}

New demands lead the meteorological information business to open up to new fields. Under the impetus of new information technology, future meteorological information service departments should have at least four important responsibilities at the same time: maintenance of meteorological services, providers of basic resources application services, managers of meteorological information security, and developers of high-quality basic meteorological data products.

\subsubsection{Maintenance of Meteorological Operational Systems}

Meteorological business is realized by meteorological business system, which is a typical information system. Whether in the current situation of vigorously implementing the intensive integration of meteorological business system or in the future of realizing the intensive integration, it is the responsibility and mission of meteorological information departments to undertake the operation and maintenance of operational system. This includes not only the system operation and maintenance in the conventional sense, but also the design of the technical framework, the formulation of specifications, the design and construction of the basic platform and the professional technical operation in the process of integration. In addition, the establishment of a complete data management system and its operation and maintenance in the form of daily business and in accordance with system norms should also be an important part of the operation and maintenance of meteorological information business in the information age.

\subsubsection{Provider of Basic Resource Application Service}

For meteorological departments, basic resources mainly include data, computing, storage, network and highperformance computing. Providing basic resources application service is the traditional business of meteorological information department and also the conventional business. Among them, providing highquality, efficient and convenient meteorological data services is the most important; not only because meteorological work is information work, cannot be separated from the guarantee of meteorological data and high-quality services; but also because in the process of the development of business system intensive trend, business process form is changing, and the sensitivity of data quality and data services is greatly enhanced; moreover, because of the development of technology, the way of business and scientific research work is changing gradually. Users are eager to change the traditional mode of downloading data locally and then analyzing and processing from the past to the mode of directly analyzing and processing and directly obtaining the analysis results at the data providers. The data service system inevitably needs to change from the original data aggregation and downloading platform to the one with more and more abundant data.

With the maturity and deep application of cloud computing, the application services of computing, storage and network resources are not only limited to providing precise flexible computing, flexible storage and SDN with cloud computing color, but also should include the cloud/client transformation of existing and existing business systems, as well as the strong specialty of distributed computing and distributed storage. Only in this way can we gradually realize the technological progress of meteorological business system in IT.

Due to the special importance of numerical prediction in meteorological operations and scientific research, the efficient application of HPC resources will become an important requirement in meteorological information operations for a long time to come. This includes not only 
the scientific and rational scheduling of HPC resources, but also the parallel processing of numerical prediction models. This is very important for improving the efficiency of business and scientific research, and for the efficient utilization of HPC resources which are still scarce resources in the next five to ten years.

\subsubsection{The Manager of Meteorological Information Security}

Information security is not only a technical problem, but also a management problem. In today's highly developed network technology, especially hacker technology, the information security work of meteorological departments must be unified management, and a unified and complete meteorological information security management system must be established. Facts have proved that a well-managed system is far safer than a technically advanced but chaotically managed system.

\subsubsection{Developer of High Quality Basic Meteorological Data Products}

Represented by global atmospheric reanalysis data and multi-source integrated precipitation/temperature products, a series of high-quality basic meteorological data products have been launched in the national meteorological information departments and have successively achieved results. The success of the research and development of these products and the gradual improvement of the research capability not only mark the significant improvement of meteorological modernization, but also meet the needs of high-quality basic data products in business and scientific research work to a large extent.

In short, the future meteorological information business, regardless of form or content, has a huge difference with the traditional business. With the introduction of new information technology, many traditional technologies will gradually disappear. However, more importantly, the change of ideas and the acceptance of new ideas, perspectives, concepts, and methods will determine the adaptability of meteorological information services to future needs.

\section{Blockchain Technology Helps the Development of Meteorological Information Technology}

Meteorological department is an information department. The basis of meteorological business is the global sharing of meteorological data. The essence of meteorological forecast is the application of meteorological data. The key of public meteorological service is the effective supply of high-efficiency and high-quality meteorological information. Information business ability is the basic support ability of meteorological business.

\subsection{Blockchain + Meteorology Big Data}

Meteorology is closely related to economic development and social life. Meteorological departments are high-tech departments, which have accumulated a large amount of data. There is no doubt that these data are valuable resources and wealth. We should fully tap the value of these resources, make good use of these resources, and provide more accurate and personalized meteorological service products that are close to people's livelihood, production, and reality.

\subsubsection{Meteorological Big Data Can Greatly Reduce Credit Cost through Blockchain}

The main application of big meteorological data in weather-related industries is to establish certain rules between the data and predict the future. According to the forecast results, the industry formulates corresponding strategies in order to maximize the benefits. Due to the trend of customization, commercialization, and diversification in the application of big meteorological data in various industries, data barrier is a real obstacle in the process of realizing big meteorological data. Every industry, like an information island, cannot achieve data fusion, let alone in-depth analysis of data. Therefore, in order to make good use of big meteorological data, we must break the "data barrier" between different industries and truly achieve data sharing, in order to achieve greater value of big meteorological data, thereby reducing disaster losses to a greater extent and creating more wealth for society. This requires strengthening trust and data sharing among meteorological data departments. To achieve this, the application of blockchain technology is the most reliable and inexpensive way. Blockchain transactions and transaction data in the future can be completely stored in the blockchain and become our personal credit; all the big data will become everyone's clear property rights credit resources, which is also the foundation of future global credit system construction.

\subsubsection{Blockchain "Patching" for Meteorological Big Data}

Big data security has always been a very important issue. With the characteristics of non-tampering and traceability, blockchain technology has attracted people's attention in recent years. It has been found that blockchain technology is of great significance to key links such as data flow and sharing. That is to say, blockchain technology solves the key problems in data flow and sharing through two ways of "stamping" and "encryption". On the one hand, the blockchain registers and authenticates the data; confirms the source, ownership, right of use and circulation path of big data assets; and makes the transaction records recognized, transparent and traceable on the whole network. Thus, data can be capitalized. Once data is generated, it will always bear the original author's "brand", which makes data more secure when it is traded as commodities or assets. On the other hand, blockchain technology can protect data from being leaked through a variety of encryption technologies. When the data is processed and placed on the blockchain, digital signature technology enables only authorized people to access the data; data privacy can be guaranteed and shared to authorized research institutions through private keys; data can be stored uniformly on the decentralized blockchain, and data analysis can be carried out without access to the original data. Protection can also be safely provided to research institutions and researchers to share. Document [10] uses "patch" to describe the role of 
blockchain technology in existing technology. The emergence of blockchains is just a patch for traditional technology, which has a good application in trust, privacy, security and so on. In recent years, intelligent terminals, cloud computing, big data, etc., have been patched all the way; blockchain is a new patch.

\subsection{Blockchain + Artificial Intelligence- Accelerator in Meteorological Field}

Blockchains and AI are considered to be the two major technological forces that change the logic of the development of the whole Internet. AI needs large amounts of data as the "feed" for robotic in-depth learning and uses big data from different fields to "train" the corresponding AI. But Internet companies with big data regard their big data resources as their core assets, and the degree of openness is not enough. In order to make the data thoroughly commercialized and socialized, we must use blockchain.

\subsubsection{Artificial Intelligence under the Feeding of Meteorological Big Data is Becoming a Powerful Assistant in Forecasting}

Artificial intelligence is empowering medical, education, safety, biology, energy and finance industries. Meteorological industry is naturally in line with the application scenario of artificial intelligence because of its rich knowledge and data accumulated over a long period of time. Artificial intelligence, by virtue of its super-strong algorithm, directly finds out the association from the complex data information and summarizes the rules because it can process the huge amount of data information that human beings cannot imagine in a very short time. From this point of view, AI can help us solve problems that are difficult or even impossible to solve in traditional ways. For example, in climate prediction, the prediction accuracy that human beings can achieve is very limited, and it is difficult to break through after reaching a certain upper limit. Artificial intelligence can help scientists identify unknown atmospheric processes; find out more interaction patterns between land, atmosphere, ocean, glacier and other circles; and judge the advantages and disadvantages of climate models so as to enhance short-and medium-term weather prediction. Under the "feeding" of high-quality meteorological data, artificial intelligence will become the most effective assistant for forecasting weather and climate.

\subsubsection{Blockchain-the Best Trusted Endorser for Data Exchange}

The essence of blockchain technology is actually a decentralized distributed account book with high transparency, traceability, and tamper-proof characteristics. Once the meteorological data is linked up, the time and content of the link are open and transparent; and once linked up, it is difficult to tamper with, the content of the chain will gradually have legal effect. Traceability and accountability provide a trust basis for data exchange and sharing among different institutions and resolve the trust problem with technology.

Artificial intelligence can help us find out more correlations in weather and climate system; and blockchain technology can exchange and share endorsement of meteorological data. The combination of these two technologies can not only improve the efficiency of international meteorological cooperation, but also break through the bottleneck of prediction to a certain extent and open up a new path for the development of meteorological cause. There is no doubt that these new technologies are becoming "accelerators" in the meteorological industry.

\section{Conclusion}

At present, a new round of scientific and technological revolution represented by information technology is in progress. Artificial intelligence, big data, cloud computing, Internet of Things, mobile Internet of Things and so on provide opportunities for the transformation and development of meteorological information. Information technology has entered a new stage of comprehensive penetration, cross-border integration, accelerating innovation and leading development, which has promoted new changes in meteorological informatization.

Artificial intelligence needs a large amount of data as the "feed" for robotic in-depth learning and uses big data from different fields to "train" the corresponding artificial intelligence. The large-scale application and sharing of big data need to be based on blockchains and make corresponding development in multi-chain, fragmentation, new intelligent contracts, and large-scale consensus. Blockchain and artificial intelligence, big data, cloud computing are effectively combined to further promote the development of meteorological information.

\section{Funding}

This research was supported by the National Natural Science Foundation of China (No. 91437112)

\section{References}

[1] Shen Wenhai, Constructing a complete Meteorological data Management system. China's information technology, 2, 82-91, Feb. 2017.

[2] Yuan yong, Wang fei-yue, Blockchain: The Satate of the Art and Future Trends. Acta Automation Sicnica, 42(4), 481-494, April 2016.

[3] YU Li'na, ZHANG Guofeng, et al, "Modern Agricultural Product Supply Chain Based on Block Chain Technology”. Transaction of the Chinese Society for Agricultural Machinery, 48(Supplement), 387-393, Dec. 2017.

[4] He Pu, Yuge, et al, "Survey on Blockchain Technology and Its Application Prospect”. Computer Science, 44(4), 1-7, Apr. 2017.

[5] Boukabara S A., E. Maddy, K. Ide, K. Garrett, E. Jones, K. Kumar, N. Shahroudi, and A. Neiss, 2018: "Exploring Using Artificial Intelligence (AI) for NWP and Situational Awareness Applications,” Austin, Texas, Amer. Meteor. Soc., Available: https://ams.confex.com/ams/98Annual/webprogram/Paper330911. html.

[6] Heye A. D., J. Cain, K. Venkatesan, A. Kommaraju, C. George, and P. Brown, 2018: "Precipitation Nowcasting Leveraging Deep Learning and HPC Systems to Optimize the Data Pipeline,” Austin, Texas, Amer. Meteor. Soc., Available: https://ams.confex.com/ams/98Annual/webprogram/Paper327679. html. 
[7] Collins W, M. Prabhat, E. Racah, Y. Liu, K. Kashinath, C. Pal, J. C. Biard, K. E. Kunkel, M. Wehner, and T. O'Brien, 2018: "Deep Learning for Detecting Extreme Weather and Climate Patterns," Austin, Texas, Amer. Meteor. Soc., Available: https://ams.confex.com/ams/98Annual/webprogram/Paper328029. html.
[8] Wang Xijin, "Analysis on the Future Application Service trend of Meteorological Big Data,” Information \& Comunications, 172(4), 290-291, 2017.

[9] Shen Wenhai, Meteorological Informatization and Management in Cloud era, Electronic industry publishing house, Aug. 2017.

[10] Gu Yan, "Block chain + Big data: 'Stamp', 'encrypt' for Data," Chinese, Observation,” 38-40. Available: http://www.cnki.net.

(C) The Author(s) 2019. This article is an open access article distributed under the terms and conditions of the Creative Commons Attribution (CC BY) license (http://creativecommons.org/licenses/by/4.0/). 